\title{
Melting and thermodynamic properties of rare gas nanocrystals
}

\author{
A.I. Karasevskii and V.V. Lubashenko \\ G.V. Kurdyumov Institute for Metal Physics, 36 Vernadsky Str., Kiev 03142, Ukraine \\ E-mail address: akaras@imp.kiev.ua \\ vilu@imp.kiev.ua
}

Received December 12, 2008

\begin{abstract}
A self-consistent statistical method [Phys. Rev. B66, 054302 (2002)] is used to describe thermodynamic properties of free rare gas nanocrystals using thin plates as examples. It is shown that size influence on thermodynamic properties of nanocrystals is caused by size-dependent quantization of the vibration spectrum affecting the parameters of a statistical distribution function of atomic displacements and, thus, governing size dependence of average values of energetic contributions to the Gibbs free energy of the system. For Xe nanocrystals, we present calculated size dependences of the Debye temperature, heat capacity, interatomic distance, melting temperature, etc.
\end{abstract}

PACS: 64.70.D- Solid-liquid transitions; 65.80.+n Thermal properties of small particles, nanocrystals, and nanotubes.

Keywords: rare gas nanocrystals, thermodynamic properties.

\section{Introduction}

The effect of size dependence of the melting temperature was discovered in the middle of the last century in electron microscopy studies of granular metallic films [1]. It was found that the melting temperature of free nanocrystals is substantially depressed as the crystal size decreases. The temperature can be changed by hundreds degrees, and this effect is pronounced even in the mesoscopic size range. Later, it was also established that this phenomenon has universal nature. It is inherent in any crystalline matter: metallic and semiconductor nanocrystals [2-5], rare gas [6,7] and ionic solids. It should be noted that depression of the melting point is observed only in free nanocrystals. If a nanoparticle is embedded into some medium, it may be considerably superheated $[8,9]$.

Recently it was reported that thermodynamic properties of nanocrystals, such as cohesion energy [10,11], Debye temperature $[10,12,13]$, activation energy of diffusion $[14,15]$, vacancy formation energy $[16,17]$ etc. display also size dependence. These experimental facts point to size influence on statistical characteristics of atoms in a nanoparticle.

In early theoretical studies of thermodynamics of nanocrystals, authors attempted to explain the strong size dependence of the melting temperature. Because of the lack of a common melting theory, these models took into account a variety of size-dependent factors affecting the transition between solid and liquid nanophases. In thermodynamic consideration of an equilibrium between these phases, an important role was ascribed to the surface of the nanoparticle $[3,5,18-20]$. Size dependence of the chemical potentials of both phases was described by introducing the capillary pressure, caused by surface curvature, and excess surface energy. Some phenomenological models of melting of free nanoparticles employed the effect of surface melting inherent in the most solids. For example, the liquid-layer model $[2,21]$ postulates formation of a quasi-liquid layer at the surface of a nanocrystal at temperature below its melting point.

Among recent theoretical approaches to description of size-dependent melting of nanocrystals, we would like to mention the liquid-drop model [22] taking into account reduction of the cohesion energy of atoms of a nanoparticle with reduction of its size and using a relation between the melting temperature and the cohesion energy [23].

A considerable advance in computational capacity made for the last decade has enabled first-principles simulation of dynamical behavior and energetic characteristics of systems consisting of more than $10^{5}$ atoms [24-32], i.e. nanocrystals in the so-called mesoscale regime. 
To our opinion, one of the most comprehensive molecular dynamics (MD) studies of thermodynamics of nanosized systems was carried out in Ref. 29 for spherical $\mathrm{Cu}$ nanocrystals. The results obtained in Ref. 29 demonstrate that both melting temperature and the latent heat of fusion decrease smoothly as particle radius decreases. The analysis of energetic and structural properties of nanocrystals revealed existence of a rather thin surface layer where average potential energy and root-mean-square (rms) displacements of atoms are largely different from those of the core region. According to results obtained in Ref. 29, the behavior of the average potential energy of atoms of a nanocrystal is characterized by essentially nonlinear rise near $T_{m}$. As shown in Refs. 33, 34 for the bulk solids, such a behavior is attributed to evolution of anharmonic instability of the phonon system of the crystal which is directly related to the melting transition.

In this work we study influence of size-dependent modification of the phonon spectrum on thermodynamic properties of rare gas nanocrystals using a thin plate of nanosized thickness as an example. Similar consideration for metallic nanoplates was carried out in Ref. 35. We show that taking into account the finite size of the nanocrystal results in quantization of its vibrational spectrum which becomes essentially discrete. Formation of a discrete vibrational spectrum affects parameters of statistical distribution of atomic displacements in the nanocrystal and, therefore, the average values of its energetic characteristics. As it will be shown, this is the chief mechanism responsible for depression of the melting point and change of thermodynamic properties of free nanocrystals with decreasing their size.

\section{Free energy of a nanocrystal}

To examine size influence on thermodynamics of nanocrystals, we use a self-consistent statistical method [36] developed to compute thermodynamic properties of simple solids. The method consists, first, in derivation of a binary distribution function of atomic coordinates and, second, in a variational procedure of computation of interatomic distance and effective parameters of quasi-elastic bonds between atoms of the crystal. It was shown that the phonon spectrum of the crystal determines parameters of the distribution function and, therefore, the average values of energetic characteristics of the crystal. Therefore, this approach allows to clarify how size-dependent modification of the phonon spectrum of a small crystal affects its thermodynamic properties. The method has been applied to description of thermal characteristics of the rare gas crystals (RGC) [36-38] and some simple fcc metals [39]. In the framework of this method we also computed the formation energy of a vacancy in the RGC as a function of temperature and demonstrated that approaching the melting point (which is assumed to be directly re- lated to the point of anharmonic instability) is accompanied by dramatic reduction of the energy required to create structure defects of the lattice $[33,34,40]$. As in our previous studies, we assume the interatomic interaction to be pairwise and approximated by the Morse potential

$$
u(r)=A\left[\mathrm{e}^{-2 \alpha\left(r-R_{0}\right)}-2 \mathrm{e}^{-\alpha\left(r-R_{0}\right)}\right] .
$$

The parameters $A, R_{0}$, and $\alpha$ for RGC have been determined in Ref. 33 so that calculated values of interatomic distance, bulk modulus, and sublimation energy at zero temperature fitted the corresponding experimental data.

In this work we restrict ourselves to the high-temperature limit when the distribution function of atomic displacements in a simple crystal may be presented as a product of one-particle Gaussian functions [33] given by

$$
f\left(\mathbf{q}_{i}\right)=C \exp \left[-\frac{\alpha^{2} c^{2} n(\tau)}{\tau} q_{i}^{2}\right],
$$

where $\mathbf{q}_{i}$ is the displacement of an atom from the $i$ th lattice site, $\tau=k_{B} T / A$ is the reduced temperature, $c$ is a dimensionless effective parameter of quasi-elastic bond of neighboring atoms, and the coefficient $n(\tau)$ determines contribution of the phonon spectrum to the distribution width. At high temperature it is expanded into a power series as [36]

$$
n(\tau)=\sum_{l=0}^{\infty}(-1)^{l} n_{l}\left(\frac{c \Lambda}{\tau}\right)^{2 l}
$$

where

$$
\Lambda=\frac{\hbar \alpha}{\sqrt{M A}}
$$

is the de Boer parameter for the Morse potential, $M$ is atomic mass. The condition for the applicability of the high-temperature approximation is given by inequality $\tau>c \Lambda$. Coefficients $n_{l}$ are determined by the phonon spectrum of the crystal [36]. For example,

$$
\begin{gathered}
n_{0}=\frac{1}{2} \sum_{j} \int \widetilde{\omega}_{j}^{2}(\mathbf{K}) \mathrm{e}_{j x}^{2}(\mathbf{K}) d \mathbf{K}, \\
n_{1}=\frac{1}{24} \sum_{j} \int \widetilde{\omega}_{j}^{4}(\mathbf{K}) \mathrm{e}_{j x}^{2} d \mathbf{K},
\end{gathered}
$$

where $\mathbf{K}$ is reduced wave vector [36],

$$
\widetilde{\omega}_{j}(\mathbf{k})=\left(\frac{M}{A \alpha^{2} c^{2}}\right)^{1 / 2} \omega_{j}(\mathbf{k})
$$

is the reduced frequency of a phonon with wave vector $\mathbf{k}$ and branch $j$, calculated from the dynamical matrix of the lattice, and $e_{j x}(\mathbf{k})$ is the projection of a phonon polariza- 
tion vector on the $x$ axis. The integration in (5) and (6) is carried out over the first Brillouin zone. For a bulk fcc crystal, $n_{0}=2, n_{1}=5 / 6$. Equations (5) and (6) were derived in Ref. 36 for the bulk crystals, but, according to Ref. 41 , such expressions are applicable to any finite system of bound harmonic oscillators.

In the high-temperature approximation the Gibbs free energy $\Phi$ of a simple fcc crystal consisting of $N$ atoms can be written as $[33,36]$

$$
\begin{aligned}
& g(\tau, p, c, b)=\frac{\Phi}{A N}=\left(\frac{\tau}{3}+3 \tau \log \frac{c \Lambda}{\tau}\right)+ \\
& +\frac{z}{2}\left[\mathrm{e}^{-2 b+\frac{2 \tau}{n(\tau) c^{2}}}-2 \mathrm{e}^{-b+\frac{\tau}{2 n(\tau) c^{2}}}\right]- \\
& -\frac{a_{3} \tau^{2}}{c^{6}}\left[\mathrm{e}^{-2 b+\frac{2 \tau}{n(\tau) c^{2}}}-\frac{1}{4} \mathrm{e}^{-b+\frac{\tau}{2 n(\tau) c^{2}}}\right]^{2}- \\
& -\kappa_{l} \frac{\varepsilon}{A}\left(\frac{\sigma}{R}\right)^{6}+p \alpha^{3} v .
\end{aligned}
$$

Here $R$ is the nearest neighbor distance, $b=\alpha\left(R-R_{0}\right)$ is a reduced lattice expansion, $z=12$ is the coordination number, $p=P /\left(\alpha^{3} A\right)$ is reduced external pressure, and $v=R^{3} / \sqrt{2}$ is volume per atom.

The first term in (8) determines the entropy part of the free energy of atomic vibrations in the crystal, the second term represents the average potential energy of interaction of neighboring atoms. The third term determines a contribution to the free energy of the crystal due to the cubic anharmonicity of collective atomic vibrations, evaluated in the second order of the perturbation theory $\left(a_{3} \approx 2.31\right.$ for the $\left.\operatorname{RGC}[33,34]\right)$. The next term takes into account the long-range attraction between atoms of the crystal, with $\varepsilon$ and $\sigma$ being the parameters of the Lennard-Jones potential and $\kappa_{l}=4.91$ for the fcc lattice [33].

\section{Vibrational spectrum of a thin plate}

To determine size contribution to the free energy of a nanocrystal of size $h(h>>R)$, we proceed from the assumption that the size-dependent modification of the vibrational spectrum is most pronounced in its long-wave part, with the wave vectors $k \sim \pi / h$ or $k R \leq 1$. Such a case corresponds to the elastic vibrations of the medium and is described by wave equations of theory of elasticity. The consideration of the size dependence of thermodynamic properties of nanocrystals will be carried out for the simplest type of nanocrystals, a thin plate. In this case, the dispersion relations have a rather simple appearance. A consistent consideration of elastic vibrations and propagation of elastic waves in plates was originally performed by Lamb [42] in the framework of general theory of elasticity.

Let us consider a plate of thickness $h$, with free surfaces parallel to the $(x, y)$ plane and the origin taken at the center of the plate. The $z$ axis is normal to the free surfaces $z= \pm h / 2$. The system is assumed to be of macroscopic size in the $(x, y)$ plane, so that vibrations can propagate as plane waves in the $x$ and $y$ directions. Note that we do not consider vibrations of the plate as a whole. The components of the stress tensor vanish at the free surfaces,

$$
\begin{aligned}
& \left.\sigma_{z x}\right|_{ \pm h / 2}=0, \\
& \left.\sigma_{z z}\right|_{ \pm h / 2}=0, \\
& \left.\sigma_{z y}\right|_{ \pm h / 2}=0 .
\end{aligned}
$$

For such a system, the displacement vector $\mathbf{u}$ can be presented as a superposition of waves of the horizontal polarization, with $u_{x}=u_{z}=0, u_{y}=u$, and waves of the vertical polarization with $u_{x} \neq 0, u_{z} \neq 0$, and $u_{y}=0$. It is important that these two types of waves do not mix at the boundaries, so that they can be considered separately (see, e.g., Refs. 42,43).

Waves of the horizontal polarization are described by ordinary wave equations for the displacement $u_{y}$,

$$
\frac{\partial^{2} u_{y}}{\partial x^{2}}+\frac{\partial^{2} u_{y}}{\partial z^{2}}+\kappa^{2} u_{y}=0
$$

where $\kappa=\omega / c_{t}, \omega$ is an eigenfrequency, $c_{t}$ is transverse sound velocity. A solution of (10) in a particular case of a plane wave propagating along the $x$ direction is given by

$$
u_{y}=C \cos (\beta z-\delta) \mathrm{e}^{i \xi x} .
$$

Parameters $\beta$ and $\delta$ are found from both the boundary conditions (9) and the symmetry reasons allowing two types of the solutions, a symmetrical one, $u_{y}(z=h / 2)=u_{y}(z=-h / 2)$, and an antisymmetrical one, $u_{y}(z=h / 2)=-u_{y}(z=-h / 2)$. These solutions lead generally to a dispersion relation given by

$$
\omega_{t}=c_{t} k_{n},
$$

with $k_{n}=\sqrt{\xi^{2}+\zeta^{2}+(n \pi / h)^{2}}, n=1,2,3, \ldots$ Here $\xi$ and $\zeta$ are, respectively, projections of the wave vector on the $x$ and $y$ axes for plane waves propagating in the $x y$ plane. The projection of the wave vector on the $z$ axis takes on discrete values,

$$
k_{z}=\frac{n \pi}{h} .
$$

In the case of waves of vertical polarization, the displacement is expressed in terms of scalar $\varphi$ and vector $\Psi$ potentials, 


$$
\mathbf{u}=\nabla \varphi+\operatorname{rot} \Psi .
$$

The potential $\Psi$ can be chosen so that $\Psi_{y}=\Psi$ and $\Psi_{x}=\Psi_{z}=0$. Then $\varphi$ and $\Psi$ satisfy the wave equations,

$$
\begin{gathered}
\Delta \varphi+k^{2} \varphi=0, \\
\Delta \Psi+\kappa^{2} \Psi=0,
\end{gathered}
$$

where $k=\omega / c_{l}, c_{l}$ is longitudinal sound velocity. The solutions of Eqs. (14), (15) are given by

$$
\begin{aligned}
& \varphi=C \cos \left(\alpha z-\delta_{l}\right) \mathrm{e}^{i \xi x}, \\
& \Psi=D \cos \left(\beta z-\delta_{t}\right) \mathrm{e}^{i \xi x},
\end{aligned}
$$

where $\alpha$ and $\beta$ are real values given by

$$
\begin{gathered}
\alpha=\sqrt{k^{2}-\xi^{2}}, \\
\beta=\sqrt{\kappa^{2}-\xi^{2}} .
\end{gathered}
$$

In terms of these notations, the boundary conditions (9) are rewritten as

$$
\begin{aligned}
& \frac{\partial \Psi}{\partial z}-\left.i p \varphi\right|_{z= \pm h / 2}=0, \\
& \frac{\partial \varphi}{\partial z}+\left.i p \Psi\right|_{z= \pm h / 2}=0,
\end{aligned}
$$

where $p=\left(\xi^{2}-\beta^{2}\right) /(2 \xi)$. Hence we get the general dispersion relation for the acoustic part of the vibrational spectrum of the nanocrystal,

$$
\tan \left(\frac{\alpha h}{2}-\delta_{l}^{0}\right) \tan \left(\frac{\beta h}{2}-\delta_{t}^{0}\right)=\frac{p^{2}}{\alpha \beta} .
$$

From the symmetry reasons, the projections of the displacement vectors $u_{x}$ and $u_{z}$ should be either symmetrical or antisymmetrical. At the symmetrical conditions

$$
\delta_{l}^{0}=m \pi, \quad \delta_{t}^{0}=(2 n+1) \pi / 2, \quad m, n=0,1,2, \ldots
$$

the dispersion relation (22) appears as

$$
\cot \frac{\alpha h}{2} \tan \frac{\beta h}{2}=-\frac{p^{2}}{\alpha \beta} .
$$

The antisymmetrical condition is specified by

$$
\delta_{l}^{0}=(2 n+1) \pi / 2, \quad \delta_{t}^{0}=m \pi, \quad m, n=0,1,2, \ldots
$$

leading to

$$
\tan \frac{\alpha h}{2} \cot \frac{\beta h}{2}=-\frac{p^{2}}{\alpha \beta} .
$$

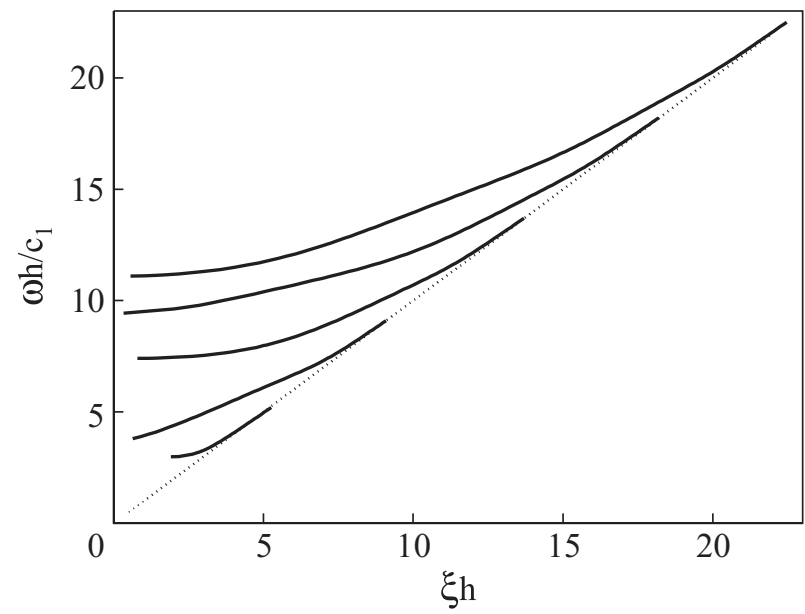

Fig. 1. Dispersion curves of longitudinal vibrations of a thin plate for $n=1, \ldots, 5$. The dotted line corresponds to the dispersion relation $\omega=c_{l} \xi$ for the bulk crystal.

Note that satisfying the symmetrical condition for $u_{x}$ implies satisfying the antisymmetrical condition for $u_{z}$, and viceversa.

In Fig. 1 we plotted dispersion curves for the longitudinal characteristic vibrations of a thin plate obtained from (23). At a rather small thickness $h$ of the plate, the long-wave part $(k R \leq 1)$ of the vibrational spectrum of the nanocrystal differs substantially from that of the bulk crystal, described by the linear relation between the frequency and the wave vector, $\omega_{j}=c_{j} \xi(j=l, t)$. The spectrum of the thin plate splits into separate vibration branches. For each branch, there is an integer number of wavelengths going across the plate thickness. The dispersion curves are well approximated by parabolas and can be analytically represented as

$$
\omega_{n}^{2}(\xi, \zeta)=\omega_{n, b}^{2}+\alpha_{n} c_{l}^{2}\left(\xi^{2}+\zeta^{2}\right)
$$

Here $\omega_{n, b}$ is the minimal value of the frequency of the $n$th vibration branch, $\alpha_{n} \approx 1$. For each of both symmetrical (23) and antisymmetrical (24) cases we have two types of solutions for $\omega_{n, b}$ corresponding to longitudinal and transverse characteristic vibrations that are generally written as

$$
\omega_{n, b}^{j} \approx \frac{\pi n}{h} c_{j},
$$

where $j=l$, $t$. As in the case of waves of the horizontal polarization (12), the wave vector for the both longitudinal and transverse standing waves of the vertical polarizations is given by Eq. (12).

A similarity of the expression for $\left(\omega_{n, b}^{j}\right)^{2}$ to the formula for the energy of a quantum particle in a one-dimensional potential well [44] is explained by the wave nature of both quantum particles and vibrational modes, exhibit- 
ing discreteness of the eigenvalues in the case of a small size of the system.

For the bulk fcc crystals, the first Brillouin zone has the form of a truncated octahedron which may be well approximated by a sphere of radius $k_{s}^{0}=\left(6 \pi^{2} / v\right)^{1 / 3}$. The radius $k_{s}^{0}$ is determined by the requirement that the number of independent values of the wave vector falling within the sphere is equal to the number of atoms of the simple lattice.

In the case of nanocrystals, evaluation of the radius $k_{s}$ of the spherical Brillouin zone requires taking into account the discrete character of $k_{z}$ in replacing summation over $\mathbf{k}$ by integration. This can be done using the Euler-Maclaurin formula [45]

$$
\sum_{k_{\min }}^{k_{\max }} f(k)=\int_{k_{\min }}^{k_{\max }} f(t) d t+\frac{1}{2}\left[f\left(k_{\min }\right)+f\left(k_{\max }\right)\right]+\ldots
$$

Then we get

$$
k_{S}=k_{S}^{0}\left(1+\frac{\pi}{4\left(k_{S}^{0} R\right)} \frac{R}{h}\right) .
$$

Presence of a size-dependent factor in (28) results in appearance of similar corrections to the coefficients $n_{l}$ in (3),

$$
n_{l}(h)=n_{l}^{0}\left(1+\gamma_{l} \frac{R}{h}\right),
$$

where $n_{l}^{0}$ is the bulk value of $n_{l}$, the first two coefficients $\gamma_{l}$ are $\gamma_{0}=0.68$ and $\gamma_{1}=0.74$. As will be shown below, the size dependence of the coefficients $n_{l}(h)$ leads to a number of physical effects observed in nanocrystals.

It should be noted that the above procedure of calculation of the coefficients $\gamma_{l}$ seems to give overestimated values, because Eq. (28) for the quasi-discrete wave vector is valid, strictly speaking, only in the range $k R \leq 1$, sensible to the particle size. Near the boundaries of the Brillouin zone, the values of $\mathbf{k}$ are determined by the structure of the crystal lattice and are slightly dependent of the crystal size. However, the structure of Eq. (28) should remain, on the whole, the same, so that $\gamma_{l}$ may be considered as parameters of the theory.

\section{Thermodynamic properties of nanocrystals}

The equilibrium values of the quasi-elastic bond parameter $c_{0}$ and reduced lattice expansion $b_{0}$ are determined from minimization of the Gibbs function (8) with respect to $c$ and $b$,

$$
\left.\frac{\partial g}{\partial c}\right|_{\tau, p, b}=0,\left.\quad \frac{\partial g}{\partial b}\right|_{\tau, p, c}=0 .
$$

The condition for the equilibrium values of the variational parameters to exist is that

$$
D=\operatorname{det}\left|\begin{array}{ll}
g_{c c}^{\prime \prime} & g_{c b}^{\prime \prime} \\
g_{b c}^{\prime \prime} & g_{b b}^{\prime \prime}
\end{array}\right|>0 .
$$

The sufficient condition $D=0$ defines the point of anharmonic instability of the crystal where the minimum of $g(\tau, p, c, b)$ with respect to $c$ and $b$ disappears.

In the case of nanocrystals, the Gibbs free energy depends, besides temperature $\tau$ and pressure $p$, on the crystal size $h$ representing an additional independent thermodynamic variable (while volume per atom is unchanged). As a result, a number of thermodynamic characteristics of the crystal display size dependence. For example, the Debye temperature is directly proportional to the equilibrium value of the parameter $c_{0}(\tau, p, h)$ and is written as $[37,38]$

$$
\Theta_{D} \approx\left(6 \sqrt{2} \pi^{2}\right)^{1 / 3}\left\langle\kappa_{j \mathbf{k}}\right\rangle A \Lambda c_{0}(\tau, p, h) / k_{B},
$$

where coefficients $\kappa_{j \mathbf{k}}=\omega_{j \mathbf{k}} /(k R)$ account for different polarizations of the acoustic waves, and $\left\langle\kappa_{j \mathbf{k}}\right\rangle \approx 0.67$ for the fcc crystals. The volume and temperature dependence of $\Theta_{D}$ determines the $\mathrm{Gr}=$ üneisen parameter,

$$
\gamma_{G}=-\frac{\left(\partial \ln \Theta_{D} / \partial \ln V\right)_{T}}{1-\left(\partial \ln \Theta_{D} / \partial \ln T\right)_{V}} .
$$

The equilibrium value of $b_{0}(\tau, p, h)$ governs the interatomic distance, the coefficient of thermal expansion, and the bulk modulus of the nanocrystal. All these properties would depend on the crystal size.

If the melting temperature $T_{m}$ is assumed to be identical to the temperature of anharmonic instability of the crystal $[33,34]$, then it can be shown in the framework of the present statistical model that $T_{m}$ is related to the factor $n(\tau)$ as

$$
\tau_{m}=\frac{k_{B} T_{m}}{A} \sim \frac{1}{a_{3} n^{3}(\tau)} .
$$

Hence it follows that even a slight increasing of $n(\tau)$ due to reduction of the crystal size results in appreciable depression of the melting temperature of the nanocrystal. Since $R / h<<1$, it follows from (34) that

$$
\frac{T_{m}}{T_{m}^{b}}=1-\frac{\beta}{3 h},
$$

where $T_{m}^{b}$ is the melting temperature of the bulk material, and $\beta=9 \gamma_{0} R$. This is the well-known relationship between the relative melting temperature of a planar nanocrystal and its inverse size (see, e.g., Ref. 22).

In Figs. 2 and 3 we plotted, respectively, the interatomic distance and the reduced coefficient of thermal expansion $\alpha=d b / d \tau$ in Xe nanocrystals versus particle size 


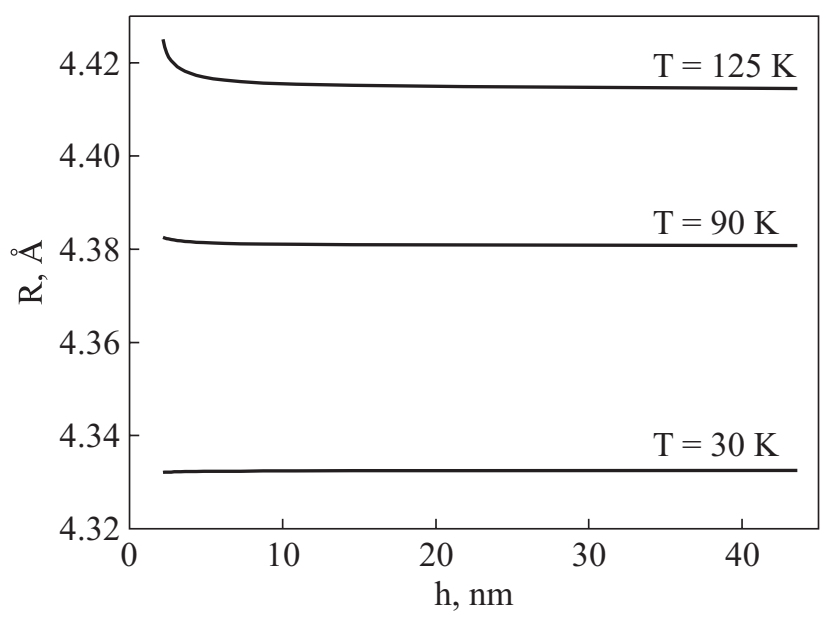

Fig. 2. Size dependence of the interatomic distance of $\mathrm{Xe}$ nanocrystals at different temperatures.

at three values of temperature calculated from (30). Hereafter all theoretical results are presented for zero external pressure. A nanocrystal of size $h \approx 2.18 \mathrm{~nm}$ melts at $T=125$ $\mathrm{K}$, which explains some nonlinear rise of $\alpha(h)$ in the top curve.

While the interatomic distances in the considered nanocrystals show no marked dependence on the crystal size, the effective parameter $c_{0}(\tau, p, h)$ of the quasi-elastic bond varies substantially with $h$. Such dependence calculated for Xe nanocrystals is illustrated with Fig. 4 which demonstrates that $c_{0}$ decreases nonlinearly as $h$ decreases even at temperatures much lower than the corresponding melting temperature of the nanocrystal.

Size dependence of the Debye temperature $\Theta_{D}$ of small crystals was observed in a number of experimental studies $[10,12,13]$. In the framework of the present approach, $\Theta_{D}$ is proportional to the quasi-elastic bond parameter $c_{0}(32)$. The $\Theta_{D}(h)$ dependence of Fig. 5 agrees

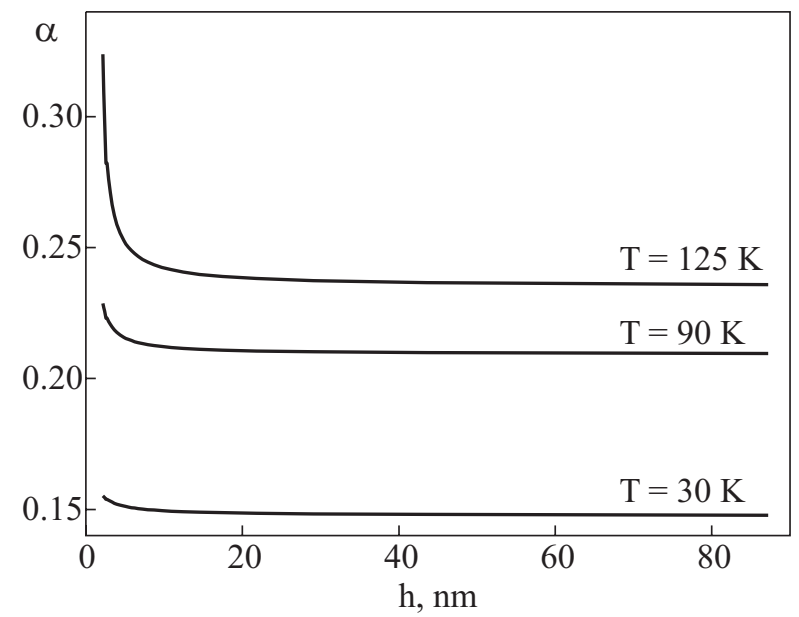

Fig. 3. Size dependence of the coefficient of thermal expansion of Xe nanocrystals at different temperatures.

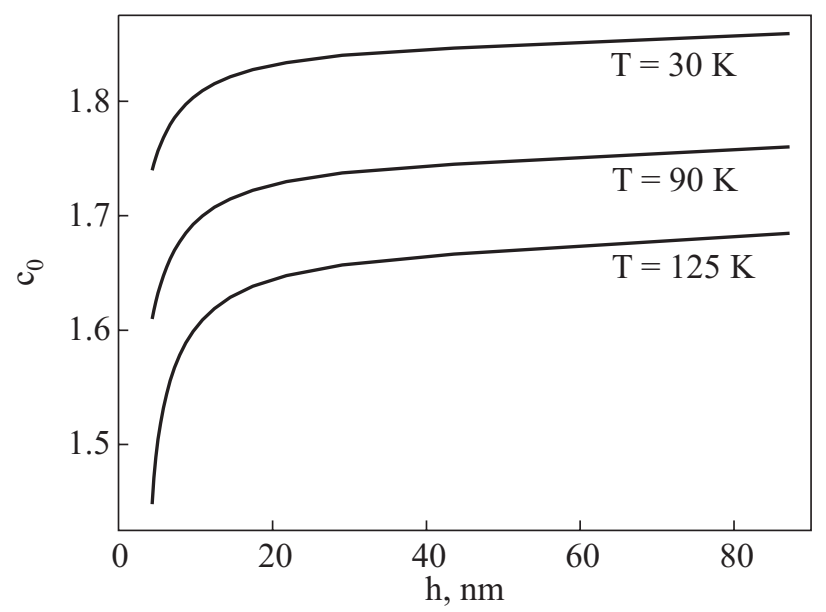

Fig. 4. Size dependence of the effective quasi-elastic bond parameter $c_{0}(\tau, h)$ of Xe nanocrystals at different temperatures.

qualitatively with that obtained experimentally, e.g., for gold nanoparticles [12].

In many bulk solids, the melting transition is preceded by anomalous behavior of temperature derivatives of thermodynamic functions (isobaric heat capacity, thermal expansion coefficient, Grüneisen parameter) due to evolution of the anharmonic instability in the phonon subsystem of the crystal as $T \rightarrow T_{m}$ [33,34]. As seen from Fig. 6, the isobaric heat capacity $C_{P}$ behaves itself in a similar way when the melting point is approached via reduction of the crystal size at constant temperature (the top curve). In other words, approaching the melting point by changing a thermodynamic variable corresponding to the size $h$ of the nanocrystal is in some way similar to variation of temperature or pressure of the crystal.

In Fig. 7 we show the melting temperature of a free Xe thin plate as a function of its thickness calculated from Eqs. (30) and the condition $D=0$, compared with that obtained from Eq. (34).

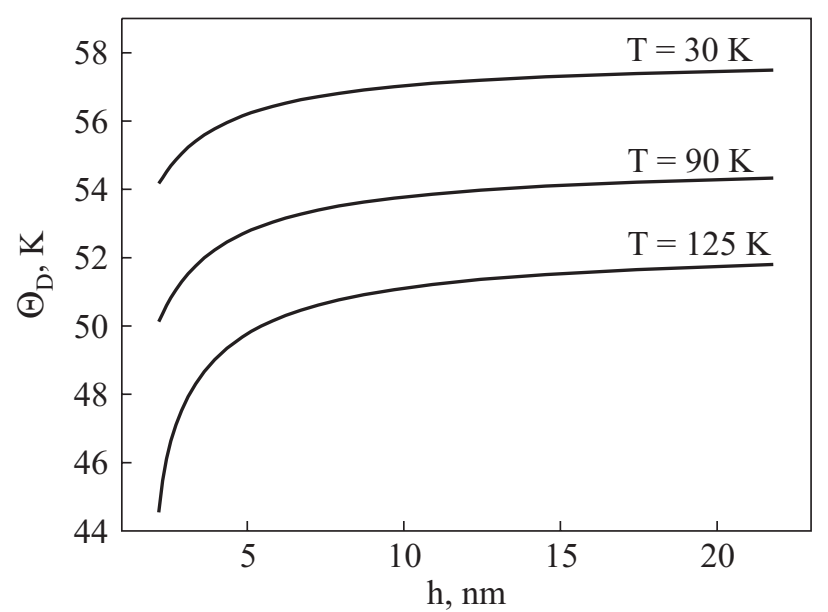

Fig. 5. Size dependence of the Debye temperature of Xe nanocrystals at different temperatures. 


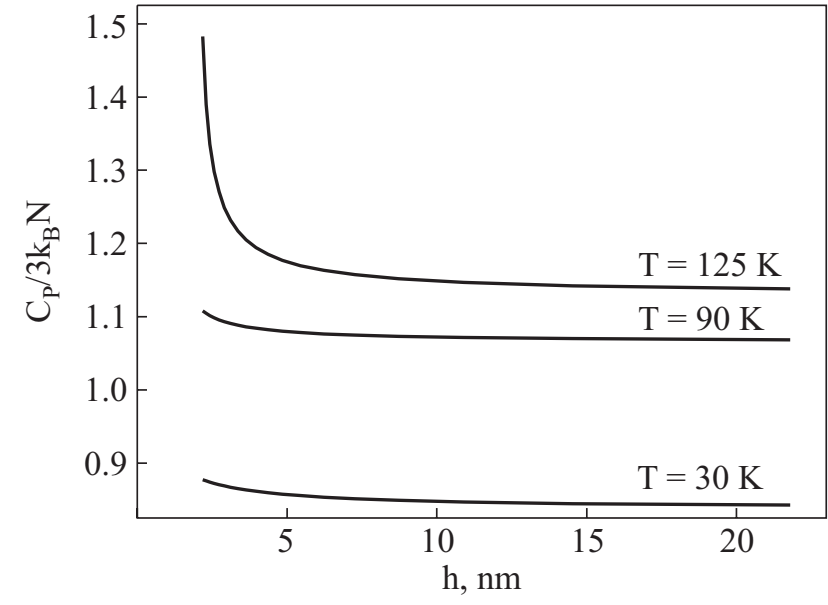

Fig. 6. Size dependence of the isobaric heat capacity of $\mathrm{Xe}$ nanocrystals at different temperatures.

At present, the only generally recognized melting criterion is the empirical Lindemann rule suggesting that a solid melts when the rms displacement of atoms reaches a characteristic fraction of the interatomic distance. In terms of notations accepted in this work, the Lindemann ratio $\delta$ is given by

$$
\delta^{2}=\frac{\left\langle q^{2}\right\rangle}{R^{2}}=\frac{3 \tau}{4 c_{0}^{2}(\tau)(\alpha R)^{2} g_{t}} .
$$

Here $g_{t} \approx 0.77$ is the correlation smearing of the distribution width of an atom at a lattice site [36].

The results presented in Figs. 4 and 8 allow us to treat the physical meaning of the Lindemann criterion in terms of the solid state instability. The evolution of the anharmonic instability is always accompanied by nonlinear reduction of the quasi-elastic bond parameter $c_{0}(\tau, p, h)$ which reaches its minimal value at the instability point corresponding either a critical temperature $\tau_{c}$ or a critical

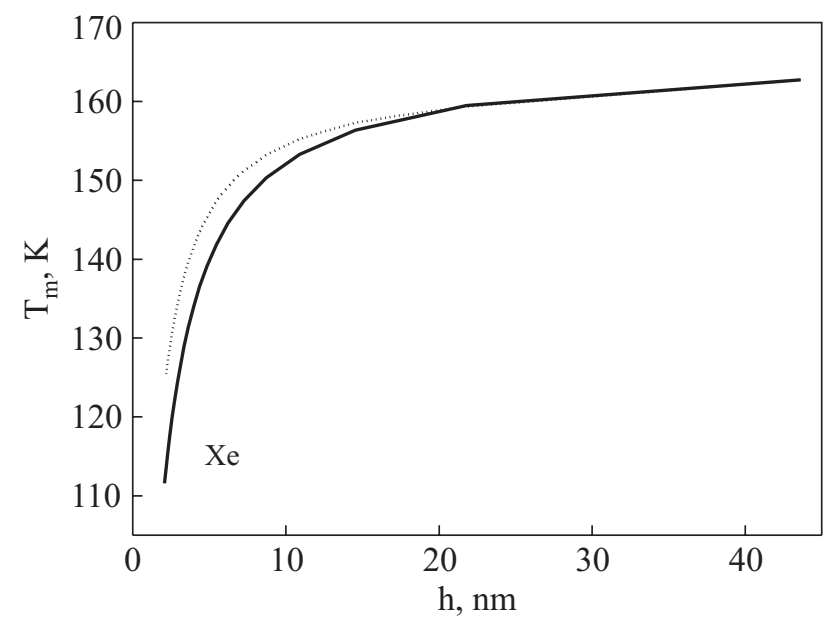

Fig. 7. Melting temperature of a free Xe thin plate versus its thickness calculated by minimization of the Gibbs free energy (8) (solid curve) and from Eq. (34) (dotted curve).

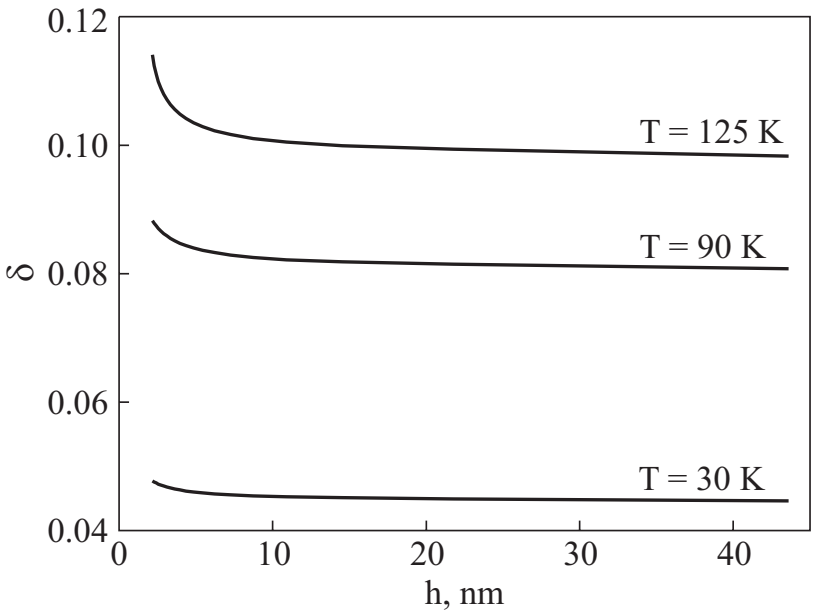

Fig. 8. The relative rms displacement of atoms (the Lindemann ratio) of Xe thin plate versus its thickness at different temperatures.

size of the particle (Fig. 4). According to (36), such a reduction of $c_{0}$ in the critical range results in dramatic increasing of the rms displacement of atoms, so that its ratio to the average interatomic distance rises up to $\delta \approx 0.1$. In addition to increasing the rms displacement, the critical range is characterized by a nonlinear rise in the isobaric heat capacity (Fig. 6), the coefficient of thermal expansion etc. as well as by a sharp drop in the formation energy of the structural lattice defects, as was shown for bulk solids $[33,34,40]$, thus promoting a transition to a structurally disordered phase.

Reduction of the crystal size involves, along with a shift of the temperature range of the anharmonic instability towards lower temperatures, a respective temperature shift of peculiarities of thermodynamic properties. This effect is illustrated in Fig. 9 presenting the temperature dependence of the Grüneisen parameter computed for different crystal sizes.

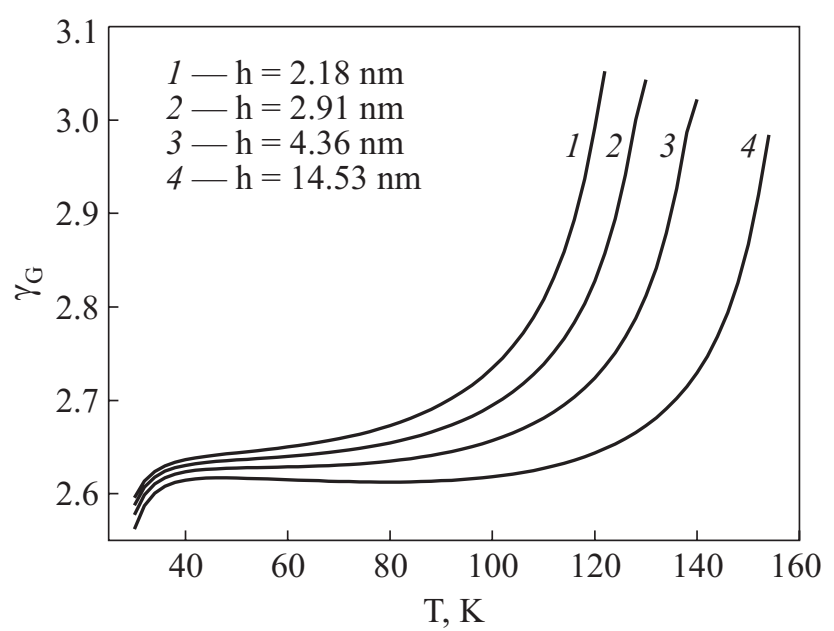

Fig. 9. The temperature dependence of the Grüneisen parameter of Xe nanocrystals of different values of thickness. 
In conclusion, one should note that influence of discreteness of the spectrum of eigenvalues on energetic characteristics of many-particle finite systems was considered previously for atomic nuclei [49] and nanoparticles of degenerated semiconductors [50]. In these studies it was demonstrated that corrections due to the discreteness of the spectrum are proportional to $h^{-1}$.

\section{Discussion}

So far there is a large amount of experimental data concerning size influence on the bulk properties of nanoparticles, such as cohesion energy [10,11], Debye temperature $[10,12,13]$, and activation energy of diffusion $[14,15]$. One of the most pronounced manifestations of change of thermodynamic properties observed in nanosized crystalline systems is the effect of reduction of the melting temperature of free nanocrystals [1-9]. So the principal problem of statistical description of thermodynamics of nanocrystals is elucidation of the mechanism of size effect on statistical characteristics of atoms in such systems. Relying on the results of MD simulations of thermodynamic properties and melting of nanocrystals $[29,30]$, it is natural to conclude that a thin (relative to the particle size) surface layer has only a minor influence upon the bulk properties of the particles; moreover, in the case of spherical particles an additional capillary pressure should contribute to increasing of both the melting temperature and the Debye temperature.

It is shown in this work that the principal size-dependent mechanism governing thermodynamic behavior of a nanocrystal is quantization of its vibrational spectrum. It leads to increasing of the parameter $n(\tau)$ of the statistical distribution function of atomic coordinates (2) and changing, as a consequence, the average value of the interaction energy of atoms in the nanocrystal. One emphasizes that such an impact on a crystalline system mediated by direct change of the statistical distribution function is inherent only in nanosystems. In this connection, it is worth noting a peculiar character of thermodynamic response of a nanocrystal to variation of its size. As the crystal's size decreases, the parameter $c_{0}(\tau, p, h)$ of quasi-elastic bond of its atoms decreases (Fig. 4), as well as the Debye temperature (32), while interatomic distance remains nearly constant (Figs. 2, 3). The melting temperature (34) of the nanocrystal is markedly decreased (Fig. 7). Independently of the crystal size, the melting transition occurs when the Lindemann criterion is satisfied (Fig. 8). Along with a size-dependent shift of the melting temperature, there is also a corresponding shift of the range of the anharmonic instability where thermodynamic properties (isobaric heat capacity, coefficient of thermal expansion etc.) display a peculiar behavior.

\section{Acknowledgement}

This work was supported in part by Award no. 28/08-H in the framework of the Complex Program of Fundamental Investigations «Nanosized systems, nanomaterials, nanotechnology» of National Academy of Sciences of Ukraine.

1. M. Takagi, J. Phys. Soc. Jpn 9. 359 (1954).

2. C.R.M. Wronski, Br. J. Appl. Phys. 18, 1731 (1967).

3. C.J. Coombes, J. Phys. F: Met. Phys. 2, 441 (1972).

4. N.T. Gladkich, R. Neidermayer, and K. Spiegel, Phys. Status Solidi 15, 181 (1966).

5. Ph. Buffat and J.-P. Borel, Phys. Rev. A13, 2287 (1976).

6. F. Celestini, R.J.-M. Pellenq, P. Bordarier, and B. Rousseau, Z. Phys. D37, 49 (1996).

7. K.K. Nanda, Chem. Phys. Lett. 419, 195 (2006).

8. H. Saka, Y. Nishikawa, and T. Imura, Philos. Mag. A57, 895 (1988).

9. L. Zhang, Z.H. Jin, L.H. Zhang, M.L. Sui, and K. Lu, Phys. Rev. Lett. 85, 1484 (2000).

10. C.C. Yang and S. Li, Phys. Rev. B75, 165413 (2007).

11. W.H. Qi and M.P. Wang, J. Mater. Sci. Lett. 21, 1743 (2002).

12. G. Kästle, H.-G. Boyen, A. Schröder, A. Plettl, and P. Ziemann, Phys. Rev. B70, 165414 (2004).

13. C.C. Yang, M.X. Xiao, W. Li, and Q. Jiang, Solid State Commun. 139, 148 (2006)

14. T. Shibita, B.A. Bunker, Z. Zhang, D. Meisel, C.F. Vardeman II, and J.D. Gezelter, J. Am. Chem. Soc. 124, 11989 (2002).

15. Q. Jiang, S.H. Zhang, and J.C. Li, J. Phys. D37, 102 (2004).

16. T. Korhonen, M.J. Puska, and R.M. Nieminen, Phys. Rev. B51, 9526 (1995).

17. C.J. Zhang and A. Alavi, J. Am. Chem. Soc. 127, 9808 (2005).

18. P. Pawlow, Z. Phys. Chem. 65, 1 (1909); ibid. 65, 545 (1909).

19. K.-J. Hanszen, Z. Phys. 157, 523 (1960).

20. P.R. Couchman and W.A. Jesser, Nature (London) 269 , 481 (1977).

21. K.F. Peters, J.B. Cohen, and Y.-W. Chung, Phys. Rev. B57, 13430 (1998).

22. K.K. Nanda, S.N. Sahu, and S.N. Behera, Phys. Rev. A66, 013208 (2002).

23. J. Tateno, Solid State Commun. 10, 61 (1972).

24. Y. Wang, S. Teitel, and Christoph Dellago, Chem. Phys. Lett. 394, 257 (2004).

25. Y. Qi, T. Cagin, W.L. Johnson, and W.A. Goddard III, J. Chem. Phys. 115, 385 (2001).

26. L. Hui, F. Pederiva, B.L. Wang, J.L. Wang, and G.H. Wang, Appl. Phys. Lett. 86, 011913 (2005).

27. U. Tartaglino, T. Zykova-Timan, F. Ercolessi, and E. Tosatti, arXiv:cond-mat / 0504680 v2: 3 Aug 2005.

28. F. Ercolessi, W. Andreoni, and E. Tosatti, Phys. Rev. Lett. 66, 911 (1991).

29. F. Delogu, Phys. Rev. B72, 205418 (2005).

30. F. Delogu, Phys. Rev. B75, 235421 (2007). 
31. S.J. Zhao, S.Q. Wang, D.Y. Cheng, and H.Q. Ye, J. Phys. Chem. B105, 12857 (2001).

32. F. Baletto and R. Ferrando, Rev. Mod. Phys. 77, 371 (2005).

33. A.I. Karasevskii and V.V. Lubashenko, Phys. Rev. B71, 012107 (2005).

34. A.I. Karasevskii, W.B. Holzapfel, and V.V. Lubashenko, J. Low Temp. Phys. 139, 609 (2005).

35. A.I. Karasevskii and V.V. Lubashenko, Eur. Phys. J. B66, 375 (2008).

36. A.I. Karasevskii and V.V. Lubashenko, Phys. Rev. B66, 054302 (2002)

37. A.I. Karasevskii and W.B. Holzapfel, Phys. Rev. B67, 224301 (2003).

38. A.I. Karasevskii and W.B. Holzapfel, Fiz. Nizk. Temp. 29, 951 (2003) [Low Temp. Phys. 29, 711 (2003)].

39. A.I. Karasevskii and V.V. Lubashenko, Phys. Status Solidi B241, 1274 (2004).

40. A.I. Karasevskii and V.V. Lubashenko, Fiz. Nizk. Temp.33, 758 (2007) [Low Temp. Phys. 33, 578 (2007)].
41. R.P. Feynmann, Statistical Mechanics, Benjamin, New York (1972)

42. H. Lamb, Proc. Math. Soc., London, 98, 205 (1920).

43. A.E.H. Love, Treatise on the Mathematical Theory of Elasticity, New York, Dover (1944).

44. L.D. Landau and E.M. Lifshitz, Quantum Mechanics, Pergamon Press, Oxford (1965).

45. E. Madelung, Mathematical Apparatus of Physics, Springer, Berlin (1957).

46. Physical acoustics. Principles and Methods, W.P. Mason (ed.), Academic Press, New York and London (1968), vol. 4, Applications to Quantum and Solid State Physics. Part B.

47. K.K. Nanda, S.N. Behera, and S.N. Sahu, J. Phys.: Condens. Matter 13, 2861 (2001).

48. W.H. Qi and M.P. Wang, J. Nanopart. Res. 7, 51 (2005).

49. S.A. Gurvits, A.A. Migdal, and A.M. Polyakov, Zh. Eksp. Teor. Fiz. 46, 213 (1964).

50. M.A. Krivoglaz and A.I. Karasevskii, Fiz. Tverd. Tela 17, 2565 (1975) [Sov. Phys. - Solid State 171709 (1976)]. 